Dermatologische Zeitschrift. 1931;62:I-IV

\title{
Contents, Vol. 62, 1931
}

\section{Inhaltsverzeichnis.}

Origizalarbeiten.

Seite Paul Linser zu seinem 60. Geburtstage am 4. September 1931. Von

Erich Schmidt 3

Beirach I. S., Eine neue Elektrode für die Ionophorese der Piostata

und der Samenbläschen 278

Bernstein, F., Überempfindlichkeit gegen Phenolphthalein 368

Boas, Kurt, Über die Alkalitherapie der geschlossenen männlichen

Harnröhrengonorrhoe 179

Böhm , A., und L. Vámos, Esophylaxie und retikuloendotheliales System 246 Busch, N.,

Morbus Pringle. Subunguale Fibromatose. Papillomatosis

cutis et mucosae. Molluscum pendulum 8

Carrie, C, siehe Schreus, H. Th.

$\mathrm{C}$ hatschaturjan, siehe Jordan, A.

Clara, Oswald, Über die Behandlung der weiblichen Gonorrhoe mit

Gkmokokken-Mischvakzine 15

Dieckmann, Fritz, Ilosacea und Rhinophym in Literatur und Kunst 20 Dittrich, 0., Lupus vulgaris serpiginosus unter dem Bilde einer

chronischen vegetierenden Pyodermie

Dobroff, siehe Jordan, A.

Edel, W., Uber die Häufigkeit der Hauttuberkulose in Württemberg 31

Eisenlohr, E., siehe Memmesheimer, Alois M.

Fauser, Marta, Gefäßwandveränderungen bei septischen Hautblutuugen 36

Finkelstein, F., siehe Ricliter, Wilhelm.

Hoffmann, Erich, Uber die Methode des Gefrierschneidens lebens-

frischer unfixierter Hautstücke nach 0. Schultz-ßrauns (ideale Biopsie) 5 Huldschinsky, Kurt, Vergleichende Beobachtungen zwischen Quarz-

lampe und Vitaluxlampe $\quad 358$

Jordan, A., Psoriasis vulgaris. Gemeinsame Arbeit von Dobroff,

Kedroff, Mentschikorf, Michailoff, Nicolaew, Popowa-Blum, Staroff, und Chatschaturjan unter Leitung von A. Jordan Moskau 221

Kedroff, siehe Jordan. A.

Kertopati, Sartono, Serologische Untersuchungen mit der Mikro-

reaktion nach Meinicke bei syphilisinfizierten und gesunden Mäusen 174

Koch, Franz, Zui · Frage der Varizenverödung nach Linser 46

Linser, Karl, und Sophie Pötschke, Umschriebene Fettnekrose

der Neugeborenen 53

Memmesheimer, Alois M. und E. Eisenlohr, Untersuchungen über

die Suggestivbehandlung der Warzen 63 
Mentschikoff, siehe Jordan, A.

Michailoff, siehe Jordan, A.

Müller, Theodor, Ein erythematüs-bullös-pustulöses Exanthem bei

Polyarthritis acuta. (Hierzu Tafel I) 269

Nast, Otto, Erste Goldversuche in der Luestherapie 69

Nékám jun., Ludwig, Ein Fall von myeloischer Leukämie mit Be-

teiligung der Haut 158

Nikolaew, siehe Jordan, A.

IV lnhaltsverzeiclmis.

Seit $\theta$ Photinos, Panagiotis B., Über Blut- und Liquorbefunde bei ge-

sunden und syphilitischen Affen 274

Pincussen, Ludwig, Uatersuchung zum Mechanismus der Ichthyol-

wirkung 258

Popowa-Blum, siehe Jordan, A.

Pötschke, Sophie, siehe Linser, Karl.

Richter, Wilhelm, und P. Finkelstein, Über die spezifische

Behandlung allergischer Hauterkratikungen 164

Schmidt, Erich, Fragen der Varizenverödung mit sklerosierenden In-

jektionen unter besonderer ßerücksiclvtigung der nach der Injektions-

behandlung beschriebenen Todesfälle $\quad 76$

Schmidt, Erich, Paul Linser zum 60. Geburtstage 3

Schreus, H. Th, und C. Carrie, Beobachtungen bei einern Fall von

kongenitaler Porphyrie 347

Schubert, Marian, und Thekla Toenges, Mikroskopische und

kulturelle Untersuchungen über gramnegative ${ }^{\wedge}$ den Gonokokken

ähnliche Diplokokken im weiblichen Urogenitaltrakt 362

S el lei, Josef, Zur Weißfleekenkrankheit, (White spot disease.) Beitrag zur

Therapie der mit der Sklerodermie in Vcrbindung stehenden Atrophien 237 Star off, siehe

Jordan, A.

Toenges, Thekla, siehe Schubert. Martin. Vámos, L., siehe Böhm, A.

Veiel, Fritz, Zur KenПtnis der Pityriasis rosea 91

Vohwinkel, K. H., Über die Alterserscheinungen des Hautbindegewebes

und über die sogenannte „Elastica mimica” bei verschiedenen Eassen 95 Zurhelle, E, Die

Aufgaben der Dermatologie und Venerologie. Rede,

gehalten zum Auftritt der ordentlic·hen Professur für Dermatologie

und Venerologie an der Reichsuniversität zu Groningen am 9. Mai 1931143

Wladimir Wladimirowitsch Iwanow $\dagger \quad 345$

Gesellschaftsberichte.

Berliner Dermatologische Gesellscliaft. Gemeinschaftliche Sitzung der

Berliner Mikrobiologischen und Dermatologischen Gesellscliaft am

4. V. $1931 \ldots 184$

Sitzung vom 12. V. $1931 \quad 184$

Sitzung vom 9. VI. 1931281

- Sitzung vom 14. VII. $1931 \quad 378$

Gemeinsame Tagung der Niederländischen Vereinigung von Dermatologen

und der Vereinigung rheinisch-westfälischer Dermatologen in Bonn. 
Sitzung am 16. und 17. V. 1931

291

Kölner Dermatologische Gesellscliaft. Sitzungen vom 24.IV. und26. VI. 1931105

- $\quad$ Sitzung vom 31. VII. 1931316

Royal Society of Medicine. Section of Dermatology. Sitzungen vom 16. X.,

20. XL und 18. XII. 1930, 15.1. 1931

108

North of England Dermatological Society. Sitzung vom 13. III. 1931 . . 119

Periodísche Literatur. Zusammengestellt von Dr. Arthur Alexander-

Charlottenburg $\quad 120,196,317,393$

Buchbesprechungen 140

Personalíen und Tagesnachrichten $\quad 142,219,346,426$

Sachregister 427

Name $\pi$ register 436 